\title{
Shear-Modulus Investigations of Monohydroxy Alcohols: Evidence for a Short-Chain- Polymer Rheological Response
}

Hecksher, Tina; Jakobsen, Bo; Dyre, J. C.; Figuli, R.; Gainaru, Catalin; Wilhelm, Manfred; Böhmer, Roland

Published in:

Physical Review Letters

DOI:

10.1103/PhysRevLett.112.098301

Publication date:

2014

Document Version

Publisher's PDF, also known as Version of record

Citation for published version (APA):

Hecksher, T., Jakobsen, B., Dyre, J. C., Figuli, R., Gainaru, C., Wilhelm, M., \& Böhmer, R. (2014). Shear-

Modulus Investigations of Monohydroxy Alcohols: Evidence for a Short-Chain-Polymer Rheological Response.

Physical Review Letters, 112(098301), [098301]. https://doi.org/10.1103/PhysRevLett.112.098301

\section{General rights}

Copyright and moral rights for the publications made accessible in the public portal are retained by the authors and/or other copyright owners and it is a condition of accessing publications that users recognise and abide by the legal requirements associated with these rights.

- Users may download and print one copy of any publication from the public portal for the purpose of private study or research.

- You may not further distribute the material or use it for any profit-making activity or commercial gain.

- You may freely distribute the URL identifying the publication in the public portal.

Take down policy

If you believe that this document breaches copyright please contact rucforsk@kb.dk providing details, and we will remove access to the work immediately and investigate your claim. 


\title{
Shear-Modulus Investigations of Monohydroxy Alcohols: Evidence for a Short-Chain-Polymer Rheological Response
}

\author{
C. Gainaru, ${ }^{1, *}$ R. Figuli, ${ }^{2}$ T. Hecksher, ${ }^{3}$ B. Jakobsen, ${ }^{3}$ J. C. Dyre, ${ }^{3}$ M. Wilhelm, ${ }^{2}$ and R. Böhmer ${ }^{1}$ \\ ${ }^{1}$ Fakultät für Physik, Technische Universität Dortmund, 44221 Dortmund, Germany \\ ${ }^{2}$ Karlsruher Institut für Technologie, 76128 Karlsruhe, Germany \\ ${ }^{3}$ DNRF Centre "Glass and Time", IMFUFA, Department of Sciences, Roskilde University, Postbox 260, DK-4000 Roskilde, Denmark
}

(Received 19 May 2013; revised manuscript received 10 June 2013; published 4 March 2014)

\begin{abstract}
Liquids composed of small-molecule monohydroxy alcohols are demonstrated to display rheological behavior typical for oligomeric chains. This observation was made possible by rheological experiments in which more than seven decades in frequency and more than five decades on the mechanical modulus scale are covered. The singly hydrogen-bonded monohydroxy alcohols were chosen because they display significant, but surprisingly poorly understood effects of intermolecular association. Based on the present shear study, one can apply theoretical concepts of polymer science to understand the anomalous physical behavior of a wide range of hydrogen-bonded liquids.
\end{abstract}

DOI: 10.1103/PhysRevLett.112.098301

PACS numbers: 82.30.Rs, 77.22.Gm, 83.60.Bc

Rheological experiments have long been thought to allow for a clear-cut distinction between simple smallmolecule liquids and macromolecular melts [1]. The dynamic viscosity of small-molecule fluids is typically governed by local flow processes reflecting short-ranged interactions. Conversely, in melts of chain molecules it is the covalently bonded backbone structure which governs the slowest relaxation of externally imposed mechanical stresses [2,3]. This leads to an increase of the overall mechanical stiffness, eventually giving rise to flow behavior typical of polymer melts. Supramolecular behavior can further be fortified by noncovalent interactions mediated by, for instance Coulombic forces, metal-ligand coordination, and hydrogen bonding, the latter, e.g., in the form of acid-base pairing [4-6]. Recently, Lou et al. studied multiply bonded donor acceptor motifs, found evidence for supramolecular polymeric liquid behavior based on nuclear magnetic resonance (NMR), dielectric relaxation spectroscopy, as well as rheological measurements [7], and compared their findings with those of the singly hydrogen-bonded monohydroxy alcohols (MAs). Albeit indications for supramolecular characteristics in MAs have been reported from NMR and dielectric studies [8], this behavior is in seeming contradiction with many data collected for these small-molecule liquids during the last five decades [9-12]. Based on shear mechanical measurements and by comparing with data of nonhydrogen-bonded smallmolecule liquids the present Letter shows that MAs indeed do exhibit a rheological signal demonstrating short-chain polymerlike aggregation of MAs. Our work paves the way for their description in terms of concepts developed for transient (living) polymers.

The MAs studied in the present work are the octanols 4-methyl-3-heptanol (4M3H) and 2-ethyl-1-hexanol $(2 \mathrm{E} 1 \mathrm{H})$ (both obtained from Sigma-Aldrich and used as received), well studied using various techniques; see, e.g., [12-16]. In Fig. 1 we present the complex shear modulus $G^{*}(\nu)=G^{\prime}(\nu)+i G^{\prime \prime}(\nu)$ of $4 \mathrm{M} 3 \mathrm{H}$, covering more than 7 decades in frequency (from $10^{-3}$ to $10^{4} \mathrm{~Hz}$ ) using a piezoelectric shear modulus gauge (PSG) technique [17]. A single-peak structure in $G^{\prime \prime}(\nu)$ is observed shifting through the frequency window, indicating the temperature dependence of the structural (or $\alpha$-) relaxation time originating from the local flow processes of this viscous liquid. At first glance, the data look unsuspicious, except that a kink appears on the low-frequency flank of the dominant peak, a feature reminiscent of a terminal mode reported to occur for a variety of short-chain polymers [18-20]. All spectra from Fig. 1 can be collapsed to a master curve, see Fig. 2(a), indicating that for $4 \mathrm{M} 3 \mathrm{H}$ time temperature superposition is obeyed for the storage component and the loss component of $G^{*}(\nu)$ [21]. Here, we used the peak frequency $\nu_{\max }$ identified with $\nu_{\alpha}$ as a scaling variable, i.e., as an effective shift factor. Figure 2(a) also includes shear data recorded using the rheometer ARES G2 (from TA Instruments) that provides mechanical excitation from 0.1 to $100 \mathrm{~Hz}$ [22]. Overall, the scaled frequency range extends over about 10 decades. For 2E1H, shear data from the rheometer and the PSG technique are combined yielding the master plot given as Fig. 2(b). Again, the $G^{*}(\nu)$ data exhibit excellent scaling behavior for all frequencies near or below the peak in $G^{\prime \prime}(\nu)$. The deviations from timetemperature superposition visible at higher frequencies for $2 \mathrm{E} 1 \mathrm{H}$ are caused by a secondary relaxation stemming from localized molecular motions [12]. The shapes of the master curves for the two MAs differ, pointing to differences in their supramolecular organization. As will be discussed below, these originate from differences in the molecular structures, sketched in Fig. 2. The figure 


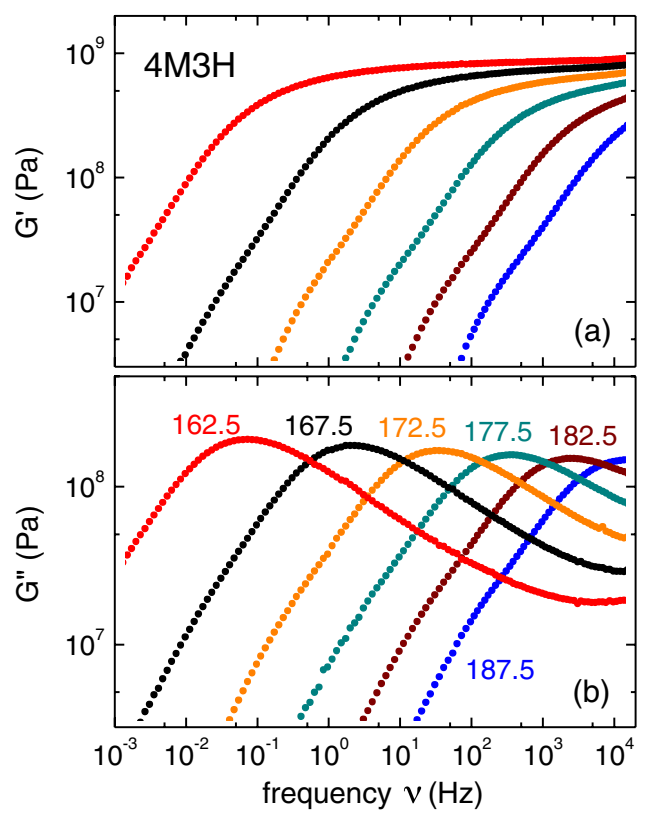

FIG. 1 (color online). (a) Real part $G^{\prime}(\nu)$ and (b) imaginary part $G^{\prime \prime}(\nu)$ of the complex shear modulus of $4 \mathrm{M} 3 \mathrm{H}$ for several temperatures (indicated in Kelvin).

illustrates that, unlike $4 \mathrm{M} 3 \mathrm{H}, 2 \mathrm{E} 1 \mathrm{H}$ features a hydroxyl group in a terminal position.

Since the focus here is on the spectral regime in which supramolecular relaxation may be expected and to facilitate detailed comparisons with the behavior of simple, nonpolymeric liquids [23], in Figs. 3(a) and (b) we show MA data in the scaled frequency range near and below $\nu_{\alpha}$. The shift factors used to scale the rheological data in Fig. 2 and Fig. 3 comply with the temperature dependent structural

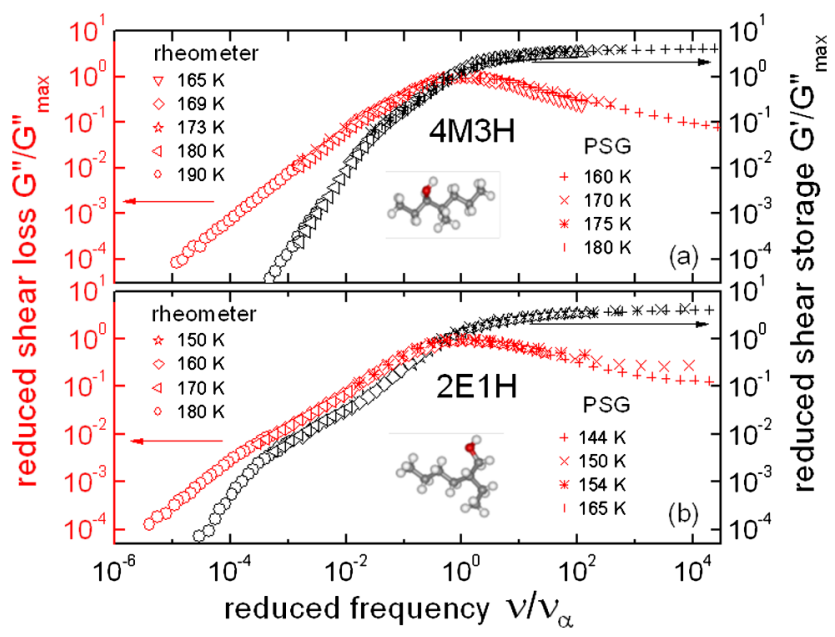

FIG. 2 (color online). Master plots for the real part (scale on the right side) and the imaginary part (scale on the left side) of $G^{*}(\nu)$ showing normalized shear moduli for (a) $4 \mathrm{M} 3 \mathrm{H}$ and (b) $2 \mathrm{E} 1 \mathrm{H}$. The data from the rheometer and from the PSG techniques as measured at the indicated temperatures agree very well. Sketches of the $4 \mathrm{M} 3 \mathrm{H}$ and $2 \mathrm{E} 1 \mathrm{H}$ molecules are also shown. relaxation frequencies determined directly from the PSG measurements; see the Arrhenius plot, Fig. 4.

In Fig. 3 we compare the $G^{*}(\nu)$ results with those for propylene carbonate and several other nonassociating liquids [23]. Overall, the main peak of their rheological spectra displays a similar shape, but clear differences are recognized on the low-frequency side of the structural relaxation peak. While the simple liquids display a unique power law in $G^{\prime \prime}(\nu)$, the shear modulus of $2 \mathrm{E} 1 \mathrm{H}$, and to some extent also of $4 \mathrm{M} 3 \mathrm{H}$, resembles normal-mode spectra of short-chain polymers $[3,18]$. Several polymerlike regimes can be distinguished in Fig. 3 from the frequency-dependent shear moduli on the low-frequency side written as $G^{\prime}(\nu) \propto \nu^{\alpha}$ and $G^{\prime \prime}(\nu) \propto \nu^{\beta}$. Generally, the
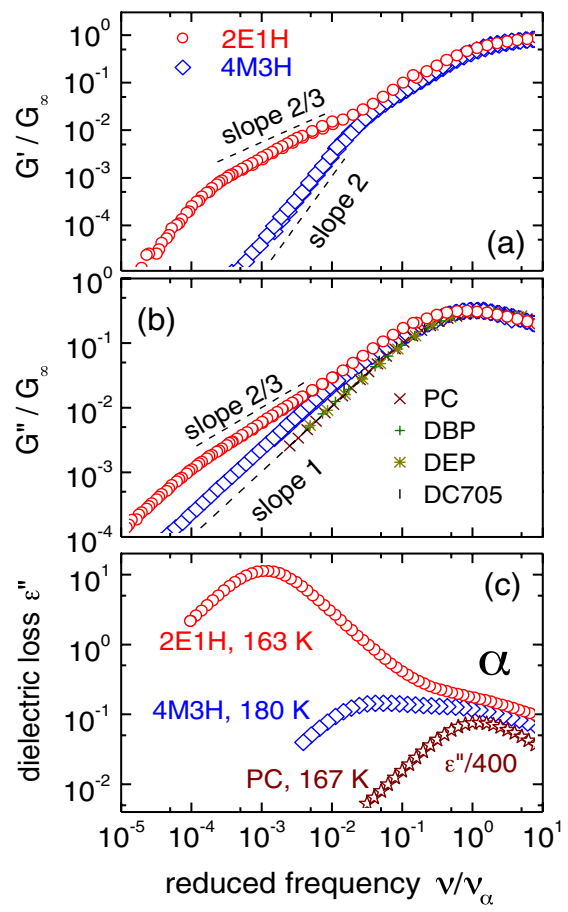

FIG. 3 (color online). (a) Real and (b) imaginary part of the reduced mechanical shear modulus as scaled by the highfrequency shear constant $G_{\infty}(\approx 1 \mathrm{GPa}$ as estimated from the current and earlier [12] broadband experiments). This plot includes moduli from the rheometer (open symbols) as well as data from Ref. [23] for liquids devoid of a supramolecular process. These latter liquids also obey time-temperature superposition [23] and comprise propylene carbonate (PC), dibutyl-phthalate (DBP), di-ethyl-phthalate (DEP), and pentaphenyl-trimethyl-trisiloxane (DC705). The dashed lines depict various power laws; see text for details. (c) Dielectric loss spectra of the alcohols $4 \mathrm{M} 3 \mathrm{H}$ and $2 \mathrm{E} 1 \mathrm{H}$. The temperature for a given system is the one at which the $\alpha$-relaxation frequency is close to $100 \mathrm{~Hz}$. Note that $G^{\prime \prime}(\nu)$ is peaked at $100 \mathrm{~Hz}$ for $154 \mathrm{~K}$ in $2 \mathrm{E} 1 \mathrm{H}$ and at $175 \mathrm{~K}$ in $4 \mathrm{M} 3 \mathrm{H}$. The dielectric strengths of the alcohols' normal modes differ significantly, whereas their $\alpha$-relaxation intensity is similar. For comparison, a loss spectrum of propylene carbonate - a typical glass former devoid of hydrogen bondingis included. 


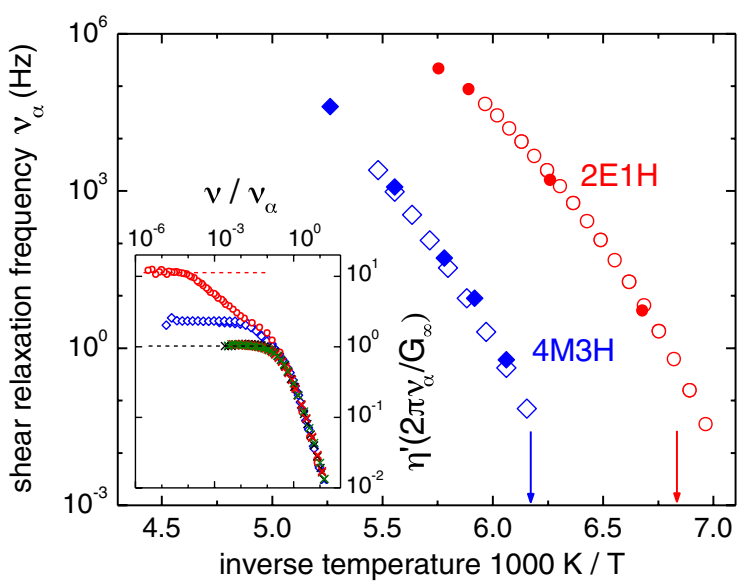

FIG. 4 (color online). Arrhenius plot of the shear relaxation frequencies. The results from the PSG experiments (open symbols) agree with those obtained by superposing the spectra measured with the high-resolution rheological setup (solid symbols). Circles refer to $2 \mathrm{E} 1 \mathrm{H}$ and squares to $4 \mathrm{M} 3 \mathrm{H}$. The inset shows $G_{\infty}$-scaled dynamic shear viscosities of the alcohols and those of small-molecule glass formers devoid of a Debye process (crosses, from Ref. [23]). For the latter liquids $2 \pi v_{\alpha} \eta^{\prime}(\nu \rightarrow 0) / G_{\infty} \approx 2 \pi \nu_{\alpha} \eta_{\alpha} / G_{\infty} \approx 1$ holds, see text, while for the alcohols a sizable low-frequency departure from this "simple-liquid" limit is obvious.

Kramers-Kronig relations imply $\alpha=\beta$; the one exception to this rule, $\alpha=2$ and $\beta=1$, marks the lowest order chain mode as observed in the low-frequency limit governed by the steady-flow viscosity. At intermediate frequencies $\alpha \approx \beta \approx 2 / 3$ - clearly obeyed for $2 \mathrm{E} 1 \mathrm{H}$-resembles the expectation of the bead-spring model including pairwise hydrodynamic interactions [24] and, consistent with this model, $G^{\prime \prime} \approx \sqrt{3} G^{\prime}$ holds here [25].

To estimate the number of molecules typically forming a supramolecular structure, let us start from the Rouse model as the simplest approach. For a polymer with steady shear viscosity $\eta_{0}$ it predicts (see p. 225 in [1]) that the slowest mode occurs at $\nu_{R}=\pi \rho R T /\left(12 \eta_{0} M_{\text {eff }}\right)$, a frequency which can be identified from the kink on the low-frequency side of the scaled modulus; see, e.g., Fig. 3. With the density $\rho$, the ideal gas constant $R$, the mass $m$ of a $2 \mathrm{E} 1 \mathrm{H}$ molecule, and using that $\eta_{0}=\lim _{\omega \rightarrow 0}\left(G^{\prime \prime} / \omega\right)=2.2 \times 10^{6}$ Pas, an effective molecular weight of $M_{\text {eff }}$ is estimated from the data of $2 \mathrm{E} 1 \mathrm{H}$ at $160 \mathrm{~K}$ [26]. Thus, $N=M_{\text {eff }} / m \approx 9$ can be interpreted as the number of segments (i.e., of $2 \mathrm{E} 1 \mathrm{H}$ molecules) within an end-to-end chain of hydroxyl groups. This $N$ is consistent with previous estimates from dielectric [8] and infrared [15] spectroscopy and with results from simulations [27] and calculations for a supramolecular polymer [7]. For $4 \mathrm{M} 3 \mathrm{H}$ the low-frequency kink is close to the segmental relaxation rate suggesting that here the relatively strong sterical shielding of the $\mathrm{OH}$ group hampers the formation of extended supramolecular structures. Their size is hard to estimate reliably but certainly they do not involve more than a few molecules.
The interpretation of our results in terms of end-to-end chains is akin to the situation in type A polymers for which along-the-contour electrical dipole moments add up to produce a pronounced dielectric normal mode [28]. Hence, it is instructive to compare the rheological results with dielectric data for the presently studied supramolecular polymers. In Fig. 3(c), dielectric loss spectra are presented, revealing that in addition to the structural relaxation a strong normal mode type of relaxation appears for $2 \mathrm{E} 1 \mathrm{H}$. In the context of MAs this feature, due to its single-exponential appearance has been called dielectric Debye relaxation [29]. For $4 \mathrm{M} 3 \mathrm{H}$ the low-frequency dielectric response is much weaker and may be affected by a ring-chain equilibrium [14], also a prominent theme in the context of supramolecular polymers [30]. Then, Fig. 3(c) demonstrates that in nonassociating nonpolymeric liquids, with propylene carbonate being an example, dielectric modes slower than the structural relaxation do not exist. This finding is fully compatible with the mechanical response of propylene carbonate and of other liquids devoid of intermolecular hydrogen bonds; cf. Fig. 3(b).

Using results from infrared, NMR, and dielectric techniques it was argued that the supramolecular structure in octanols develops an increased tendency to disintegrate thermally in the high-frequency regime $[15,16]$. Thus, in the sub-GHz range, detection of supramolecular features in MAs required the application of high-resolution ultrasonic techniques [31]. Brillouin scattering that probes mechanical response at even higher frequencies did not display a significant signature of supramolecular phenomena [11].

The contribution of the "normal mode rigidity" or "Debye rigidity" to the frequency-dependent shear viscosity of the MAs is obtained from $\eta^{\prime}(\nu)=G^{\prime \prime}(\nu) /(2 \pi \nu)$. This relation follows from the definitions of the dynamic shear viscosity and modulus. For the $\alpha$ relaxation we may write $\eta_{\alpha} \approx G_{\infty} /\left(2 \pi \nu_{\alpha}\right)$. As the inset of Fig. 4 reveals, for $2 \mathrm{E} 1 \mathrm{H}$ the viscosity increase $\Delta \eta_{D} / \eta$ or the rigidity enhancement $\Delta G_{D} / G_{\infty}$ due to the supramolecular (Debye or normal mode) process amounts to a factor of $\sim 10$. However, the polymerlike effects show up at moduli about 3 orders of magnitude lower than $G_{\infty}$, much below the detection limits of typical shear mechanical [12] and ultrasonic experiments [9]. The suprasegmental mechanical contribution observed for $2 \mathrm{E} 1 \mathrm{H}$ is comparable to that of short-chain polymers [3], but has been detected at temperatures much lower than usually applied in studies of covalently bonded polymers. Furthermore, the slower the dielectric normal mode is with respect to the $\alpha$ response, the larger also is the separation of the two modes in the mechanical spectra, and the lower will be the modulus at which the unambiguous $G^{\prime}(\nu) \propto \nu^{\alpha}$ or $G^{\prime \prime}(\nu) \propto \nu^{\beta}$ low-frequency signature of the terminal chain mode shows up. Thus, on the basis of the current work, we predict that small-molecule liquids comprising a single intermolecular peptide-related bond, such as the secondary amides $[32,33]$ for which so far only an intermediate power 
law was resolved in mechanical studies [23], also do display end-to-end chain modes.

To summarize, we identified MA as short-chain polymeric liquids. In contrast to typical supramolecular polymers, in MAs the transient-chain association is sustained via only a single intermolecular hydrogen bond. As a consequence, the observation of a chain mode in MAs becomes possible at temperatures as low as $145 \mathrm{~K}$ and, thus, very much lower than usually considered in the study of polymeric liquids. Furthermore, our findings show that MAs and predictably many other chain-forming hydrogenbonded or peptide-bonded liquids can be analyzed theoretically in terms of polymer concepts and possibly by suitably adapted approaches developed for related soft matter systems [34].

Support of this project by the Deutsche Forschungsgemeinschaft under Grant No. BO1301/8-2 is gratefully acknowledged. The centre for viscous liquid dynamics "Glass and Time" is sponsored by the Danish National Research Foundation's Grant No. DNRF61.

*Corresponding author.

catalin.gainaru@uni-dortmund.de

[1] J. D. Ferry, Viscoelastic Properties of Polymers (Wiley, New York, 1980).

[2] F. Tanaka and S. F. Edwards, Macromolecules 25, 1516 (1992).

[3] C. M. Roland, Viscoelastic Behavior of Rubbery Materials (Oxford Univeristy Press, Oxford, 2011); K. L. Ngai, Relaxation and Diffusion in Complex Systems (Springer, Berlin, 2011).

[4] For reviews, see A. Ciferri, Supramolecular Polymers (CRC Press, Boca Raton, FL, 2005); T. F. A. De Greef, M. M. J. Smulders, M. Wolffs, A. P. H. J. Schenning, R. P. Sijbesma, and E. W. Meijer, Chem. Rev. 109, 5687 (2009).

[5] S. J. George, A. Ajayaghosh, P. Jonkheijm, A. P. H. J. Schenning, and E. W. Meijer, Angew. Chem. 116, 3504 (2004); A. Ajayaghosh, R. Varghese, V. K. Praveen, and S. Mahesh, Angew. Chem. 118, 3339 (2006).

[6] J. Cortese, C. Soulié-Ziakovic, S. Tebcé-Girault, and L. Leibler, J. Am. Chem. Soc. 134, 3671 (2012).

[7] N. Lou, Y. Wang, X. Li, H. Li, P. Wang, C. Wesdemiotis, A. P. Sokolov, and H. Xiong, Macromolecules 46, 3160 (2013).

[8] C. Gainaru, R. Meier, S. Schildmann, C. Lederle, W. Hiller, E. A. Rössler, and R. Böhmer, Phys. Rev. Lett. 105, 258303 (2010).

[9] T. A. Litovitz and G. E. McDuffie, J. Chem. Phys. 39, 729 (1963).

[10] G. P. Johari and W. Dannhauser, J. Chem. Phys. 51, 1626 (1969).

[11] C. Hansen, F. Stickel, T. Berger, R. Richert, and E. W. Fischer, J. Chem. Phys. 107, 1086 (1997).
[12] B. Jakobsen, C. Maggi, T. Christensen, and J. C. Dyre, J. Chem. Phys. 129, 184502 (2008).

[13] W. Dannhauser, J. Chem. Phys. 48, 1918 (1968).

[14] L. P. Singh and R. Richert, Phys. Rev. Lett. 109, 167802 (2012), and references cited therein.

[15] C. Gainaru, S. Kastner, F. Mayr, P. Lunkenheimer, S. Schildmann, H. J. Weber, W. Hiller, A. Loidl, and R. Böhmer, Phys. Rev. Lett. 107, 118304 (2011).

[16] S. Bauer, K. Burlafinger, C. Gainaru, P. Lunkenheimer, W. Hiller, A. Loidl, and R. Böhmer, J. Chem. Phys. 138, 094505 (2013).

[17] T. Christensen, N. B. Olsen, Rev. Sci. Instrum. 66, 5019 (1995).

[18] R. W. Gray, G. Harrison, and J. Lamb, Proc. R. Soc. London 356, 77 (1977).

[19] Y. Ding, V. N. Novikov, A. P. Sokolov, R. Casalini, and C. M. Roland, Macromolecules 37, 9273 (2004).

[20] M. Paluch, S. Pawlus, A. P. Sokolov, and K. L. Ngai, Macromolecules 43, 3103 (2010).

[21] In the temperature range covered by the present study, nearinfrared investigations indicate that the $\mathrm{H}$ bond concentration changes by only about $5 \%-6 \%$ for $2 \mathrm{E} 1 \mathrm{H}[14]$ and $4 \mathrm{M} 3 \mathrm{H}$ [15], thereby not affecting time-temperature superposeability in a significant way.

[22] K. Hyun, S. Höfl, S. Kahle, and M. Wilhelm, J. Non-Newtonian Fluid Mech. 160, 93 (2009).

[23] C. Maggi, B. Jakobsen, T. Christensen, N. B. Olsen, and J. C. Dyre, J. Phys. Chem. B, 112,16320 (2008); C. Gainaru, T. Hecksher, N. B. Olsen, R. Böhmer and J. C. Dyre, J. Chem. Phys. 137, 064508 (2012).

[24] B. H. Zimm, J. Chem. Phys. 24, 269 (1956).

[25] For the Zimm model, see Fig. 9-8(b) on p. 190 and Eqs. (32) and (33) on p. 192 of Ref. [1].

[26] Here we used $\rho=0.833 \times \mathrm{g} / \mathrm{cm}^{3}, m=2.16 \times 10^{-22} \mathrm{~g}$ or $130.2 \mathrm{~g} / \mathrm{mol}$, and $\nu_{R} \approx 0.13 \mathrm{~Hz}$ at $160 \mathrm{~K}$. According to the Zimm model the terminal rate is larger than $\nu_{R}$, thus $M_{\text {eff }}$ would turn out somewhat larger if calculated using that approach. In any case, the given estimates have to be considered with some caution, because neither the Zimm nor the Rouse model address aspects characteristic for transient hydrogen-bonded chains such as exchange processes between bonded and nonbonded molecules.

[27] P. Sillrén, J. Bielecki, J. Mattsson, L. Börjesson, and A. Matic, J. Chem. Phys. 136, 094514 (2012).

[28] G. Williams, Macromol. Symp. 286, 1 (2009).

[29] P. Debye, Verh. Dtsch. Phys. Ges. 15, 777 (1913);

[30] T. Xiao, X. Feng, S. Ye, Y. Guan, S.-L. Li, Q. Wang, Y. Ji, D. Zhu, X. Hu, C. Lin, Y. Pan, and L. Wang, Macromolecules 45, 9585 (2012).

[31] R. Behrends and U. Kaatze, J. Phys. Chem. A 105, 5829 (2001).

[32] L.-M. Wang and R. Richert, J. Chem. Phys. 123, 054516 (2005).

[33] R. Ludwig, O. Reis, R. Winter, F. Weinhold, and T. C. Farrar, J. Phys. Chem. B 102, 9312 (1998).

[34] See, e.g., R. Granek and M. E. Cates, J. Chem. Phys. 96, 4758 (1992), and references cited therein. 